\title{
EDITORIAL
}

\section{WHY ARE WE STILL NEGLECTED? A CONCERN OF SENIOR PHYSIOTHERAPISTS}

Physiotherapy is regarded as one of the most lucrative and white collar professions in western world. Relieving the pain by magical skills and rehabilitation of patients are among the half marks of physiotherapy. Physiotherapists are contributing in many different domains; either it is an outpatient department, a home management program, a fitness centers or as crucial as an ICU. American Physiotherapy Association APTA divides us into almost 24 sub- specialties of this field where orthopedics, neurology, general medicine, general surgery, gynecology, pediatric, health and wellness, geriatrics and much more. Physiotherapist are the people working day and night under the catastrophic conditions hence considered as lifesavers. Patients suffering from catastrophic and epidemic disasters like earthquakes, floods, bomb blasts, and burns.

The field of physiotherapy was introduced in Pakistan in 1956, but unfortunately the significance of physiotherapy is yet to be recognized. The lack of awareness of early referral to physiotherapy and rehabilitation departments are causing adverse effects to the patients leading to permanent disabilities with each passing day in Pakistan. Physiotherapist are versatile professionals either to work with obese individuals in the health and fitness centers or in the fields with national and international sportsmen training and even working with acute injuries. Hence increasing the demands of physiotherapist around the world.

On the other hand, the patients are also following the conventional route of pain management by medicines and surgeries. Being unaware of the least invasive techniques offered in the field of physiotherapy where different exercises and techniques almost eliminate their symptoms non-surgically. These exercises and techniques include simple exercises, ultrasonic waves and short wave radiations, manual therapy and manipulation, mechanical devices (traction) and physical agents (heat, cold and electricity). Physiotherapists work with individuals to prevent the loss of mobility. Before, it occurs by developing fitness and wellness-oriented programs for healthier and more active lifestyles, providing services to individuals and populations to develop maintain and restore maximum movement and functional ability throughout the lifespan. This includes providing therapeutic treatment in circumstances where movement and function are threatened by aging, injury, disease or environmental factors. Functional movement which requires needs to be healthy.

In addition, the implications of physiotherapy in medical and health sciences are of great importance. However, the government is not paying required attention to promote this emerging field. New graduates of physiotherapy are facing difficulties in finding jobs, as the available jobs are of extremely low wedges. Consequently, forcing them to migrate from Pakistan and find their livings in the Middle Eastern or Western countries deteriorating the integrity of the noble profession. These regions not only pay well but also consider physiotherapists as medical professionals rather than paramedical staff. The government should take an initiative to save this profession by creating a recognized council for physiotherapy across the country. Moreover, job market for physiotherapists should be created by the federal and provincial governments. In order to overcome the challenges, government should facilitate the professionals of this field to influence the policy makers for a better future of the profession. Furthermore, the regulatory bodies need to establish a strong policy, and standards of physiotherapy education and profession for a better education, service and good health outcome in Pakistan.

Therefore, the expected solution for the present situation is to create awareness among common people and even medical and health related personnel about physiotherapy. Rehabilitation must be encouraged through social media, electronic media, and print media. Similarly, conducting seminars and workshops emphasizing the need for physiotherapy in Pakistan will also leave a significant impact to create awareness. Furthermore, to improve the health outcomes, it is important that physiotherapists have the best available education and training to work in health care system.

\section{Dr. Fareeda Shaheen Shah}

Consultant Physiotherapist

H.O.D Sindh Government Hospital

Saudabad, Karachi, Pakistan 